\title{
Confirmation of asthma in an era of overdiagnosis
}

\author{
V.P. Luks*, K.L. Vandemheen ${ }^{\#}$ and S.D. Aaron ${ }^{\#}$
}

ABSTRACT: It was recently shown that $30 \%$ of adults with a physician diagnosis of asthma did not have asthma when objectively assessed using a four-step algorithm involving serial spirometry, bronchial challenge testing and subsequent tapering of asthma medications. The objective of the present study was to determine how many steps in the algorithm were required in order to confirm asthma, and whether any patient-related variables were associated with earlier asthma confirmation.

A total of 540 subjects with a previous physician diagnosis of asthma were randomly recruited from the community. The number of subjects confirmed with asthma at each study visit was calculated. Regression analysis was used to determine variables associated with earlier asthma confirmation.

Of the 499 subjects who completed the diagnostic algorithm, 346 (69\%) had asthma confirmed and 150 (30\%) had asthma excluded. Of subjects in whom asthma was confirmed, including those using regular asthma controlling medications, $>90 \%$ were confirmed with only one or two study visits, by either pre- and post-bronchodilator spirometry or a single bronchial challenge test. Only $46(9 \%)$ out of 499 subjects required tapering of asthma medications and repeated bronchial challenge tests for exclusion or confirmation of asthma. Lower forced expiratory volume in $1 \mathrm{~s}$ and younger age were associated with earlier asthma confirmation.

For the majority with a previous physician diagnosis of asthma, only pre- and postbronchodilator spirometry and a single methacholine challenge test are required in order to confirm asthma.

KEYWORDS: Asthma, bronchial hyperreactivity, diagnosis, spirometry

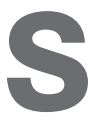
ince the late 1970s, there has been a dramatic increase in the incidence and prevalence of asthma in North America $[1,2]$. However, it is unclear whether the increased incidence of new asthma diagnoses in developed countries is appropriate, or whether asthma is being overdiagnosed in developed countries due to an increased awareness of asthma amongst healthcare providers and patients [3]. A study was recently conducted to investigate the proportion of Canadian adults who have an incorrect diagnosis of asthma [4]. A total of 540 randomly recruited subjects from the community underwent a stepwise algorithm that included up to four visits to the pulmonary function laboratory in an attempt to rule in, or rule out, physiological evidence of asthma. It was concluded that $30 \%$ of patients with a previous physician diagnosis of asthma did not have asthma when assessed objectively [4].
Currently patients diagnosed with asthma retain their diagnosis for a lifetime. No validated protocols exist to confirm or exclude asthma in patients with a previous physician diagnosis of asthma (who may or may not have been correctly diagnosed initially). Confirmation of asthma may be more difficult in patients who are taking regular asthma-controlling medications. Patients on inhaled corticosteroids, even for $<3$ months, can experience not only an improvement in symptoms but also a decrease in demonstrable airway responsiveness, even returning to the normal range on bronchial challenge testing [5]. Once started on an inhaled corticosteroid, negative bronchial challenge testing results or absence of change in postbronchodilator forced expiratory volume in $1 \mathrm{~s}$ (FEV1) may indicate either a well controlled asthmatic or a nonasthmatic. Even amongst subjects not started on inhaled corticosteroids, confirming a physician diagnosis of asthma can be
AFFILIATIONS

*University of Ottawa, and

\# The Ottawa Health Research Institute, University of Ottawa, Ottawa, ON, Canada.

CORRESPONDENCE

S.D. Aaron

The Ottawa Hospital

General Campus

501 Smyth Road

Ottawa

ON K1H 8L6

Canada

E-mail: saaron@ohri.ca

Received

Oct 182009

Accepted after revision:

Jan 052010

First published online:

Jan 142010 
difficult if there are minimal symptoms, and hence probably minimal airway inflammation, at the time of testing.

Given the associated costs of pharmacological therapy for asthma, and the previously demonstrated overdiagnosis of asthma, developing a strategy to confirm or exclude asthma in patients with a previous physician diagnosis of asthma is important. The ideal algorithm would be both sensitive and specific, and would minimise the number of visits or tests, thereby minimising cost and inconvenience to the patient. Since no previously validated algorithm to accomplish this exists, this secondary analysis was conducted in order to determine how many steps in the asthma diagnostic algorithm were genuinely required in order to definitively confirm or exclude asthma in subjects with a previous physician diagnosis of asthma. A second objective was to determine whether or not there were any patient-related factors associated with earlier confirmation of asthma.

\section{METHODS}

\section{Study population}

The study population consisted of 540 subjects recruited from across Canada by random-digit dialling between December 2005 and December 2007, and is the same cohort of subjects described by AARON et al. [4]. Inclusion criteria included: an age of $>15$ yrs; and current asthma diagnosed by a physician (method of diagnosis not specified, and proof of diagnosis provided only by the subject's account). Exclusion criteria included: subjects taking long-term oral corticosteroids; inability to undergo bronchial challenge testing due to other medical conditions; a cigarette smoking history of >10 pack-yrs; inability to undergo spirometry; and inability to provide consent [4].

\section{Asthma assessment algorithm}

The protocol to confirm or exclude asthma involved one to four patient visits to the pulmonary function laboratory [4], as outlined in figure 1 . The first visit consisted of pre- and postbronchodilator spirometry. If the patient showed an improvement in FEV1 of $\geqslant 200 \mathrm{~mL}$ and $\geqslant 15 \%$ after the bronchodilator was given, then asthma was confirmed and no further testing was required. If spirometry was negative, the patient returned for a bronchial challenge test with methacholine (MCT) at visit 2. If the MCT at visit 2 was positive (i.e. revealed a provocative concentration of methacholine causing a $20 \%$ fall in FEV1 (PC20) of $\leqslant 8 \mathrm{mg} \cdot \mathrm{mL}^{-1}$ ), then asthma was confirmed. If the MCT at visit 2 was negative, asthma was excluded in subjects not taking any asthma-controlling medications, such as leukotriene antagonists or inhaled corticosteroids, on a regular basis.

Those subjects who were taking such medications on a regular basis and gave negative test results at visits 1 and 2 were required to taper their asthma medications and undergo repeat bronchial challenge testing. After visit 2, leukotriene antagonists were discontinued and the dose of inhaled corticosteroids was halved. The subjects then returned 2-3 weeks later for visit 3, which consisted of another MCT. If the MCT at visit 3 was positive, asthma was confirmed, and, if not, long-acting $\beta$ agonists and inhaled corticosteroids were discontinued completely and the subjects returned in 2-3 weeks for visit 4 , a final MCT. Patients with a positive MCT at visit 4 had asthma confirmed. Asthma was also confirmed in those who suffered from an asthma exacerbation during the medication taper and evaluation period. Asthma was ruled out in patients who showed no evidence of acute worsening of asthma symptoms, reversible airflow obstruction or bronchial hyperresponsiveness at any visit, despite being completely weaned from asthma medications. It should be noted that short-acting $\beta$ agonists were permitted at any time during the study algorithm, but were withheld for $8 \mathrm{~h}$ prior to a MCT. Prior to discontinuation at visit 3 , long-acting $\beta$-agonists were permitted at any time, but withheld for $48 \mathrm{~h}$ prior to a MCT.

\section{Safety assessment}

Subjects in whom asthma was excluded were asked to remain off asthma medications. These subjects were followed every 2 months for a period of 6 months to determine whether they had restarted any asthma medications, had made urgent visits to a healthcare provider or an emergency department, required a hospital admission for respiratory symptoms or required systemic corticosteroids.

\section{Statistical analysis}

The number of subjects who were confirmed or excluded at each visit was calculated and then univariable analysis performed to examine whether there were any patient-related variables associated with earlier or later confirmation of asthma. These patient-specific variables were pre-selected based on previously described associations between these variables and bronchial responsiveness, and included: age, sex, smoking status, baseline percentage predicted FEV1, use of regular controlling medications, and baseline asthma symptoms based on responses to the European Community Respiratory Health Study Questionnaire (ECRHSQ). An unadjusted Chi-squared test was used to compare timing of confirmation of asthma based on the presence of these variables. A multivariable analysis was also performed using logistic regression to examine patient-related factors associated with earlier confirmation of asthma, which included all of the variables listed above.

\section{RESULTS}

Of the 540 participants in the study, 499 completed all of the study assessments and could be evaluated for a diagnosis of asthma. Table 1 shows the baseline characteristics of these patients. Three participants showed evidence of lung restriction, with a baseline FEV1 that was $<60 \%$ pred. Since these three participants were unable to safely undergo a bronchial challenge test, they were categorised as unable to classify. Of the remaining 496 subjects who completed the algorithm, 346 $(70 \%)$ had asthma confirmed and 150 (30\%) had asthma excluded. Figure 2 shows a breakdown of when each subject was confirmed or excluded according to the study algorithm.

\section{Subjects in whom asthma was confirmed}

Of the 346 subjects with confirmed asthma, 329 (95\%) had their diagnosis of asthma confirmed within two visits (fig. 2; table 2). Of the 346 subjects in whom asthma was confirmed, $164(47 \%)$ were using regular asthma-controlling medications. In those subjects taking daily anti-inflammatory asthma medications, 90\% were confirmed within two visits (147 out of 164; 90\%). All $182(100 \%)$ subjects not taking regular 


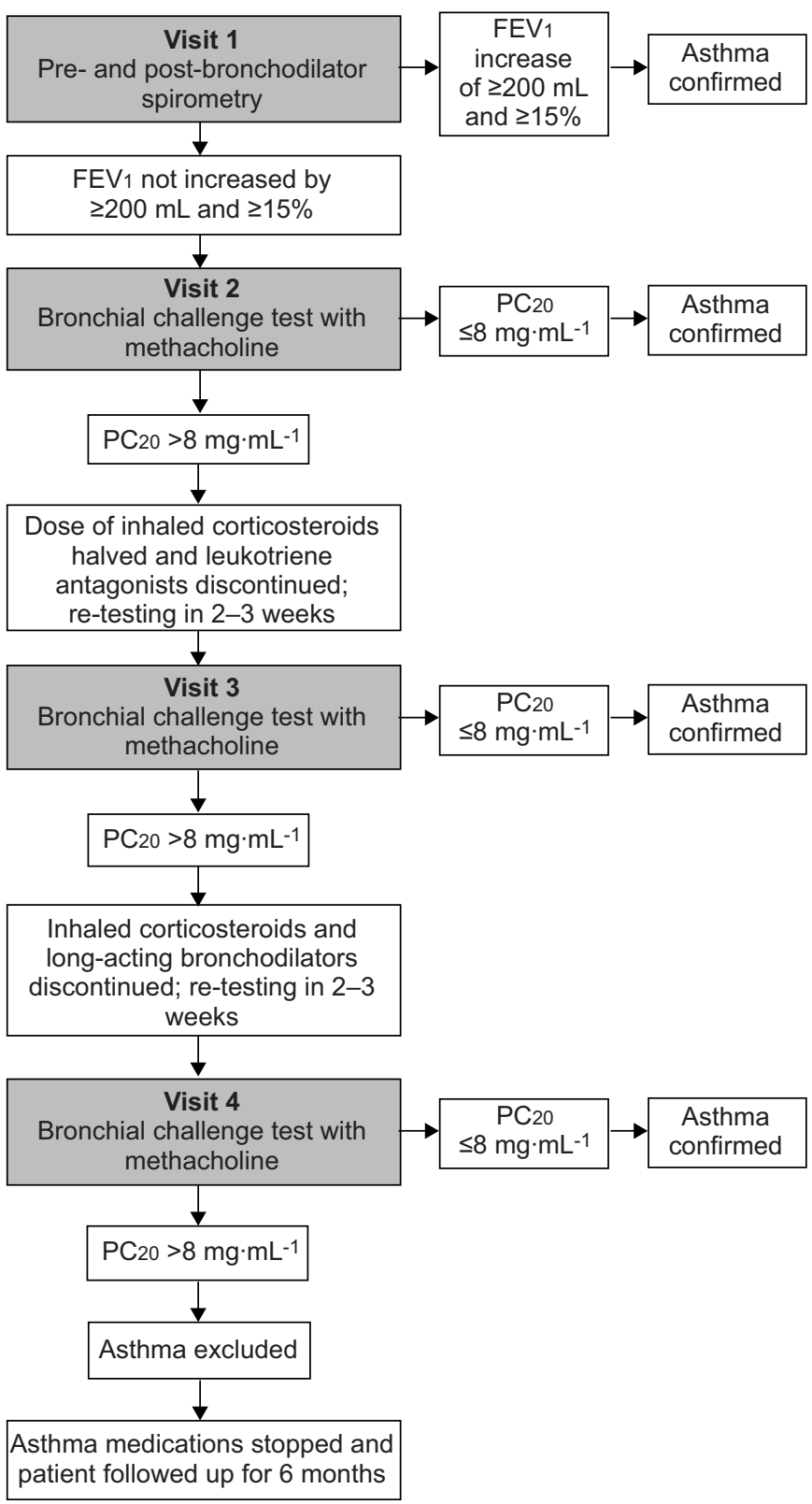

FIGURE 1. Serial asthma testing algorithm. FEV1: forced expiratory volume in 1 s; PC20: provocative concentration of methacholine causing a $20 \%$ fall in FEV1.

controlling medications were confirmed within two visits; this was expected since, as per the study protocol, only two visits (one visit for pre- and post-bronchodilator spirometry and one for a bronchial challenge test) were required in order to confirm or exclude asthma in those not using regular inhaled steroid or anti-leukotriene medications.

Univariable analysis showed no difference in the timing of confirmation of asthma between males and females, younger versus older subjects, subjects with more versus fewer respiratory symptoms based on the ECRHSQ or subjects who were obese versus those with a normal body mass index. Smokers were more likely to be confirmed earlier with asthma, and

\begin{tabular}{|c|c|c|}
\hline \multirow[t]{2}{*}{ TABLE 1} & \multirow[b]{2}{*}{ On medication ${ }^{\#}$} & \multirow[b]{2}{*}{ Not on medication ${ }^{\#}$} \\
\hline & & \\
\hline Subjects $n$ & 198 & 301 \\
\hline Age yrs & $48.2 \pm 16.3$ & $42.2 \pm 15.6$ \\
\hline Females & $137(69.2)$ & $199(66.1)$ \\
\hline Smokers & $11(5.6)$ & $27(9.0)$ \\
\hline Baseline FEV 1 \% pred & $86.2 \pm 18.8$ & $92.5 \pm 15.9$ \\
\hline Disease duration yrs & $19.9 \pm 15.5$ & $17.0 \pm 12.9$ \\
\hline BMI $\mathbf{k g} \cdot \mathrm{m}^{-2}$ & $30.2 \pm 8.5$ & $29.3 \pm 7.4$ \\
\hline \multicolumn{3}{|l|}{ Baseline symptoms } \\
\hline Wheeze & $61(30.8)$ & $82(27.2)$ \\
\hline Cough & $95(48.0)$ & $131(43.5)$ \\
\hline Shortness of breath & $76(38.4)$ & $107(35.5)$ \\
\hline Chest tightness & $61(30.8)$ & $72(23.9)$ \\
\hline Sputum production & 87 (43.9) & $96(31.9)$ \\
\hline \multicolumn{3}{|l|}{ Regular medications } \\
\hline Leukotriene antagonists & $25(12.6)$ & $0(0.0)$ \\
\hline ICS & $90(45.5)$ & $0(0.0)$ \\
\hline ICS/LABA & $106(53.5)$ & $0(0.0)$ \\
\hline
\end{tabular}

many more smokers exhibited significant improvements in bronchodilator responsiveness at visit 1 compared to nonsmokers (table 3). Similarly, subjects with a baseline FEV1 of $\leqslant 80 \%$ pred had asthma confirmed earlier than those with a baseline FEV1 of $>80 \%$ pred (table 3 ).

Subjects taking regular controlling medications required more visits to the pulmonary function laboratory in order to confirm, or exclude, a diagnosis of asthma $(\mathrm{p}<0.001)$. This difference was partly due to the algorithm design, which ensured that subjects not taking regular controlling medications were discharged from the study with a confirmation or exclusion of asthma by visit 2 . However, those subjects who were using regular asthma controlling medication, and who did not show significant bronchodilator reversibility or a positive bronchial challenge test while on medications, had to undergo medication tapering with subsequent revisits for further bronchial challenge tests.

Multivariable analysis (table 4) revealed that better lung function (higher percentage predicted FEV1) and older age, both treated as continuous variables, were significantly associated with a greater likelihood of patients requiring more than two visits to the pulmonary function laboratory in order to confirm a diagnosis of asthma. Although nonsmokers tended to require more visits to confirm asthma, this was nonsignificant in the multivariable analysis. Use of regular controlling asthma medications was not included as a variable in the multivariable analysis since only those patients using regular controlling medications required third or fourth visits to confirm asthma (as per the algorithm). 


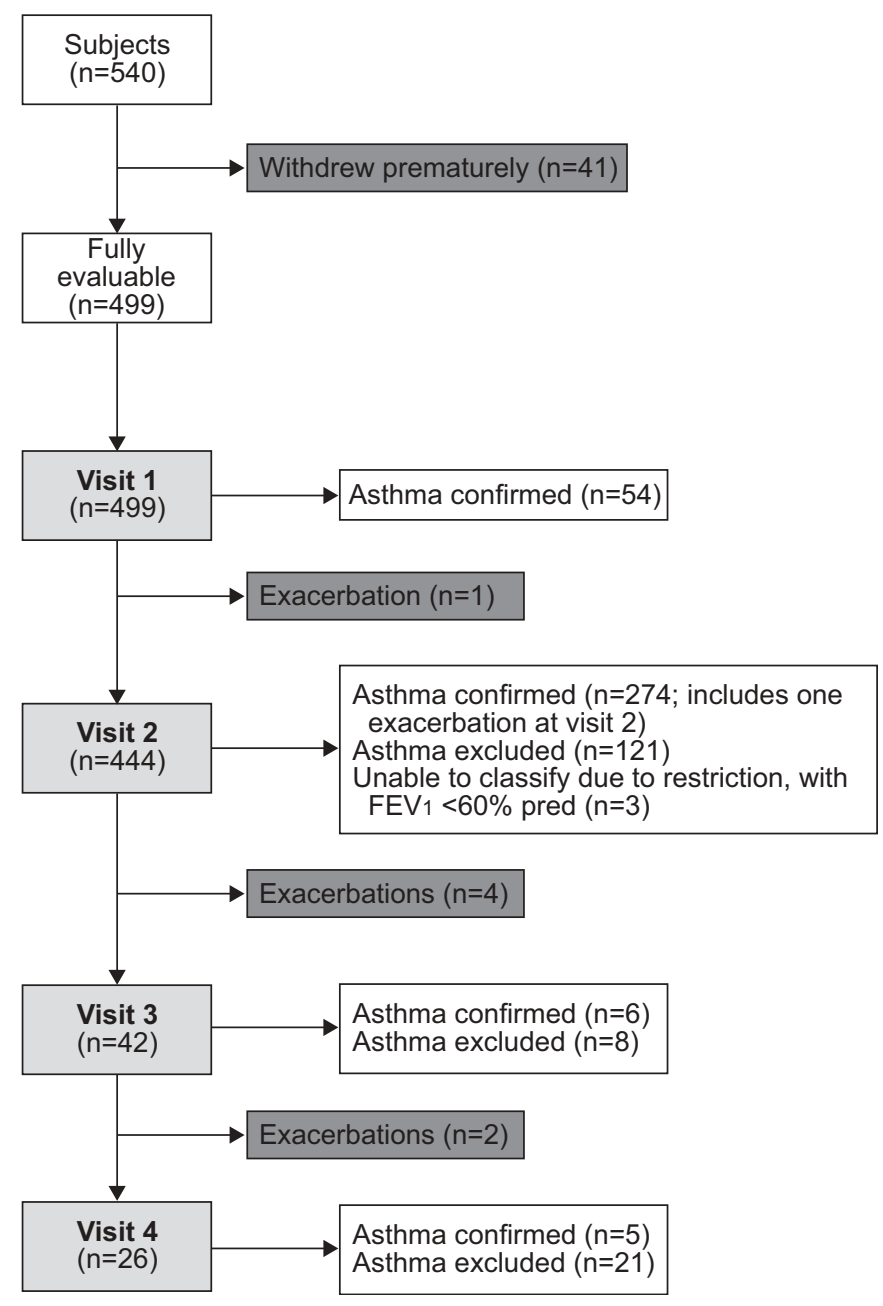

FIGURE 2. Timing of confirmation or exclusion of asthma in study subjects. FEV1: forced expiratory volume in $1 \mathrm{~s}$; \% pred: \% predicted.

\section{Subjects in whom asthma was excluded}

Asthma was excluded in 150 (30\%) out of 499 subjects. The timing of exclusion of asthma was driven by the algorithm. For example, all subjects who were not on regular controlling medications, and who had asthma excluded, were excluded at visit 2 as per the algorithm. Similarly, subjects taking regular controlling medications could only be excluded after tapering of all medications, a process which required all four visits.

\section{Safety and follow-up}

Among the 499 patients who completed the study algorithm, there were a total of only eight asthma exacerbations, two prior to tapering asthma medications, four after the subjects' dose of inhaled steroids was halved, and two after inhaled steroids and other asthma medications were discontinued completely.

Eight of the 150 patients in whom asthma was excluded were lost to follow-up. Of the 142 patients who completed follow-up, $93(66 \%)$ did not need to take asthma medications and did not require care for respiratory symptoms during the follow-up period. The remaining 49 (34\%) resumed taking an asthma medication at some point during the follow-up period; however, 17 only used bronchodilators and 12 used asthma medications for $<2$ weeks. Of the 142 subjects, 11 (7.7\%) made unplanned visits to a physician because of respiratory symptoms; two of these 11 patients received oral corticosteroid therapy.

\section{DISCUSSION}

It was found that $>90 \%$ of patients previously diagnosed with asthma, even those taking asthma-controlling medications on a regular basis, can have a diagnosis of asthma confirmed (if they truly have asthma) using pre- and post-bronchodilator spirometry and a single MCT. This was unexpected given studies showing the prolonged effect of inhaled corticosteroids on dampening bronchial responsiveness [6]. Patients taking regular inhaled corticosteroids exhibited a mean FEV1 of $86.2 \%$ pred at the start of the study. It is thus possible that patients in the present study still had relatively poor asthma control despite using regular inhaled corticosteroids, and this may explain why it was possible to show bronchodilator responsiveness and bronchial hyperresponsiveness relatively easily.

Despite the fact that the majority of subjects on regular controlling medications were confirmed without tapering of their medications, $\sim 10 \%$ of participants could not be confirmed while continuing regular anti-inflammatory asthma medications. Of this group, a third were found to have asthma once their medications were tapered and bronchial challenge testing was repeated. Although applicable to only a small minority of those taking regular controlling asthma medications, this underscores the need to taper asthma medications and repeat bronchial challenge testing if test results for asthma are initially negative in this group.

The small number of exacerbations and paucity of adverse respiratory outcomes over 6 months of follow-up (two courses of oral steroids) suggest that the present protocol is safe. Two-thirds of the exacerbations that occurred during inhaled steroid tapering occurred after the doses of inhaled corticosteroids were halved, indicating that tapering is probably safer than abrupt discontinuation of controlling medications.

\begin{tabular}{|c|c|c|c|c|c|c|}
\hline On medication & 164 & $23(14.0)$ & $124(75.6)$ & $10(6.1)$ & $7(4.3)$ & \multirow{2}{*}{$<0.001$} \\
\hline Not on medication & 182 & $31(17.0)$ & $151(83.3)$ & $0(0)$ & $0(0)$ & \\
\hline
\end{tabular}

Data are presented as $n(\%)$, unless otherwise stated. ${ }^{\#}$ : comparison between groups; ${ }^{\bullet}$ : regular asthma-controlling medications 
TABLE 3 Effects of smoking and lung function on timing of confirmation of asthma

\begin{tabular}{|c|c|c|c|c|c|c|}
\hline & Subjects $\mathrm{n}$ & Visit 1 & Visit 2 & Visit 3 & Visit 4 & p-value ${ }^{\#}$ \\
\hline Smoker & 113 & $25(22.1)$ & 85 (75.2) & $3(2.7)$ & $0(0)$ & 0.006 \\
\hline FEV $1>80 \%$ pred & 234 & $16(6.8)$ & 205 (87.6) & $8(3.4)$ & $5(2.1)$ & \multirow{2}{*}{$<0.0001$} \\
\hline FEV $1 \leqslant 80 \%$ pred & 112 & $38(33.9)$ & $70(62.5)$ & $2(1.7)$ & $2(1.7)$ & \\
\hline
\end{tabular}

Data are presented as $n(\%)$, unless otherwise stated. FEV1: forced expiratory volume in $1 \mathrm{~s} ; \%$ pred: \% predicted. ${ }^{\#}$ : comparison between groups.

In the present study, a lower FEV1 at baseline ( $\leqslant 80 \%$ pred) was associated with earlier confirmation of asthma. This is consistent with previous studies showing increased bronchial hyperresponsiveness in subjects with a lower FEV1 [7]. The lower the FEV1 at baseline the less the absolute decrease in FEV1 required to give a $20 \%$ decline following administration of methacholine [8]. In addition, lower lung function may be associated with more inflammation of the airways and hence greater bronchial hyperresponsiveness. It is not clear whether central as opposed to peripheral deposition of inhaled methacholine contributes to bronchial hyperresponsiveness in those with a lower baseline FEV1. Central particle deposition occurs in all subjects during bronchoprovocation testing and is not an important determinant of responsiveness in those with normal baseline spirometric results [9].

Younger age was also associated with earlier confirmation of asthma in the present study. Changes in bronchial responsiveness with age are inconsistent, and different studies have demonstrated increased, decreased or no change in bronchial responsiveness with ageing [10]. The effects of longer duration of disease and longer exposure to air pollutants and/or smoking readily confound associations made between bronchial responsiveness and age. Known geometric changes to the lung that occur with ageing, such as enlargement of airspaces, resulting in decreased airway traction and hence reduced airway calibre are logical explanations for an increase in bronchial responsiveness with age [10]. It is important to note that many studies examining the relationship between bronchial hyperresponsiveness and age are cross-sectional studies

\begin{tabular}{lll} 
TABLE 4 & $\begin{array}{l}\text { Multivariable analysis: patient characteristics } \\
\text { predicting requirement for multiple visits to } \\
\text { confirm asthma }\end{array}$ \\
& Multivariate OR (95\% Cl) & p-value \\
\hline Age & $1.04(1.00-1.07)$ & 0.03 \\
Smoking & $0.38(0.10-1.38)$ & 0.14 \\
Female sex & $0.60(0.21-1.74)$ & 0.35 \\
FEV1 \% pred & $1.04(1.01-1.07)$ & 0.02 \\
Obesity & $0.57(0.20-1.62)$ & 0.29 \\
ECRHSQ score & $1.44(0.50-4.13)$ & 0.50 \\
\hline
\end{tabular}

FEV1: forced expiratory volume in $1 \mathrm{~s}$; \% pred: \% predicted; ECRHSQ: European Community Respiratory Health Study Questionnaire, maximum score 5. ${ }^{\#}$ : body mass index of $\geqslant 30 \mathrm{~kg} \cdot \mathrm{m}^{-2}$. examining a general population, and it is possible that asthmatics exhibit a different chronological time course from the general population in their bronchial responsiveness. CUTTITTA et al. [11] looked at younger versus older asthmatics with similar duration of disease and baseline lung function and found no difference in bronchial hyperresponsiveness. In the present study, older participants were more likely to be taking daily inhaled corticosteroids, and this may be one reason why older age was associated with later confirmation of asthma. Additionally, it has been shown that elderly subjects show reduced responsiveness to $\beta$-adrenergic agonists [12], perhaps reducing the sensitivity of the pre- and postbronchodilator test at visit 1 .

Smoking is also well known to increase bronchial responsiveness [7]. The present univariable analysis showed earlier confirmation of asthma in smokers, although the association became insignificant in the multivariable analysis. Smokers in the present study happened to be slightly younger and exhibited more respiratory symptoms; thus the effect of smoking may have become insignificant when those other factors were taken into account in the multivariable analysis.

Past observations that females show greater bronchial responsiveness than males may be related to females having smaller lung size or airway calibre [7], or to an interaction between airway responsiveness and smoking in females that is not seen in males [13]. The present study results are consistent with either hypothesis as no evidence was found of a sex-dependent difference in the timing of asthma confirmation when accounting for both smoking status and baseline percentage predicted FEV1.

The major limitation of the present study is the fact that there is no absolute gold standard to apply in order to determine whether or not the final classification of each patient (asthma confirmed or asthma excluded) was correct. The patients in whom asthma was excluded were followed for 6 months however, it is possible for asthmatics to be symptom-free and show minimal airway inflammation and hyperresponsiveness for an unknown duration of time, especially if their asthma becomes manifest only with particular exposures. In a study of patients with undiagnosed respiratory symptoms possibly consistent with asthma who were followed for 6 months after a MCT, the sensitivity of the MCT, although far superior to either peak expiratory flow variability or postbronchodilator change in FEV1, was still only $86 \%$, allowing for the possibility of false negative results [14]. It is also possible that asthma was falsely confirmed in some patients. It is well known that bronchial hyperresponsiveness can 
occur in nonasthmatic patients, who might have been included in the present study, such as those with allergic rhinitis, those who have recently had a viral respiratory infection or smokers with normal lung function [15]. However, using a $\mathrm{PC} 20$ of $\leqslant 8 \mathrm{mg} \cdot \mathrm{mL}^{-1}$, the value used in the present study, GOLDSTEIN et al. [14] demonstrated a specificity of $100 \%$ for the MCT.

In summary, the present study has shown that $>90 \%$ of subjects who report physician-diagnosed asthma, even those who are taking regular asthma-controlling medications, can have their diagnosis of asthma confirmed with only two testing visits to the pulmonary function laboratory. For the majority of such patients, asthma medication wash-out prior to bronchial challenge testing is not necessary for confirmation of a diagnosis of asthma.

\section{SUPPORT STATEMENT}

This study was supported by the Canadian Institutes of Health Research (Ottawa, ON, Canada).

\section{STATEMENT OF INTEREST}

A statement of interest for S.D. Aaron can be found at www.erj. ersjournals.com $/ \mathrm{misc} /$ statements.dtl

\section{ACKNOWLEDGEMENTS}

The authors would like to recognise S. Doucette (The Ottawa Health Research Institute, University of Ottawa, Ottawa, ON, Canada) for collaboration on the statistical analysis used in the present study.

\section{REFERENCES}

1 National Centre for Health Statistics, Vital Statistics of the United States, 1999. Hyattsville, US Department for Health and Human Services, 1999.

2 Bai T, Kenney A, Van Gorder B. Asthma. In: Stewart P, et al., eds. Respiratory Disease in Canada. Ottawa, Canadian Institute for Health Information, Canadian Lung Association, Health Canada, Statistics Canada, 2001; pp. 33-44.
3 Eder W, Ege MJ, von Mutius E. The asthma epidemic. N Engl J Med 2006; 355: 2226-2235.

4 Aaron SD, Vandemheen KL, Boulet LP, et al. Overdiagnosis of asthma in obese and nonobese adults. CMAJ 2008; 179: 1121-1131.

5 Juniper EF, Kline PA, Vanzieleghem MA, et al. Effect of long-term treatment with an inhaled corticosteroid (budesonide) on airway hyperresponsiveness and clinical asthma in nonsteroid-dependent asthmatics. Am Rev Respir Dis 1990; 142: 832-836.

6 Juniper EF, Kline PA, Vanzieleghem MA, et al. Reduction of budesonide after a year of increased use: a randomized controlled trial to evaluate whether improvement in airway responsiveness and clinical asthma are maintained. J Allergy Clin Immunol 1991 87: 483-489.

7 Schwartz J, Schindler C, Zemp E, et al. Predictors of methacholine responsiveness in a general population. Chest 2002; 122: 812-820.

8 Apter AD, Weiss ST. Asthma: epidemiology. In: Fishman AP, ed Fishman's Pulmonary Diseases and Disorders. Vol. 1. 4th Edn. New York, McGraw-Hill, 2008; pp. 789-790.

9 O'Riordan TG, Walser L, Smaldone GC. Changing patterns of aerosol deposition during methacholine bronchoprovocation. Chest 1993; 103: 1385-1389.

10 Scichilone N, Messina M, Battaglia S, et al. Airway hyperresponsiveness in the elderly: prevalence and clinical implications. Eur Respir J 2005; 25: 364-375.

11 Cuttitta G, Cibella F, Bellia V, et al. Changes in FVC during methacholine-induced bronchoconstriction in elderly patients with asthma. Chest 2001; 119: 1685-1690.

12 Bellia V, Cibella F, Cuttitta G, et al. Effect of age upon airway obstruction and reversibility in adult asthmatics. Chest 1998; 114: 1336-1342.

13 Leynaert B, Bousquet J, Henry C, et al. Is bronchial responsiveness more frequent in women than men? A population-based study. Am J Respir Crit Care Med 1997; 156: 1413-1420.

14 Goldstein MF, Veza BA, Dunsky EH, et al. Comparisons of peak diurnal expiratory flow, post-bronchodilator FEV1 responses, and methacholine inhalation challenges in the evaluation of suspected asthma. Chest 2001; 119: 1001-1010.

15 Crapo RO, Casaburi R, Coates AL, et al. Guidelines for methacholine and exercise challenge testing - 1999. Am J Respir Crit Care Med 2000; 161: 309-328. 